\title{
Measurement of Liver and Spleen Interstitial Volume in Patients with Systemic Amyloid Light-Chain Amyloidosis using Equilibrium Contrast CT
}

Authors: Yeung J1ㄴ Sivarajan $\mathrm{S}^{1}$, Treibel $\mathrm{TA}^{2,3}$, Rosmini $\mathrm{S}^{2}$, Fontana $\mathrm{M}^{4}$, Gillmore $\mathrm{JD}^{4}$, Hawkins $\mathrm{PN}^{4}$, Punwani S1, Moon JC2,3, Taylor SA ${ }^{1}$, Bandula S1

1) Centre for Medical Imaging, University College London, 250 Euston Road, London, NW1 2PG, UK

2) Institute of Cardiovascular Science, University College London, London, WC1E 6BT, UK

3) Barts Heart Centre, St Bartholomew's Hospital, West Smithfield, London, EC1A 7BE, UK.

4) Centre for Amyloidosis and Acute Phase Proteins and National Amyloidosis Centre, Royal Free Campus, University College London, Rowland Hill Street, London, NW3 2PF, UK

\section{Corresponding author:}

Jason Yeung

$+44-7849209837$

j.yeung88@hotmail.co.uk

ORCID: 0000-0003-2415-4824

\section{Keywords:}

Tomography, X-ray Computed; Contrast Media; Amyloidosis; Radiographic Image Interpretation, Computer Assisted; Extracellular Space

\section{Compliance with Ethical Standards}

Conflict of interest: The authors declare that they have no conflict of interest.

Funding: This study has received funding from the National Amyloid Centre to cover scanning costs. 


\section{Acknowledgements:}

We gratefully acknowledge the contributions of CT radiographers Elaine Atkins and Preeya Patel at University College London Hospital, David Edwards at the Royal Free Hospital, and Toshiba's CT specialists Mark Condron and Tristan Lawton.

TA Treibel and S Bandula are supported by Doctoral Research Fellowships from NIHR, UK (NIHR-DRF-2013-06-102/NIHR-DRF-2011-04-008). S Taylor is an NIHR senior investigator. The majority of this work was undertaken at University College London Hospital and University College London, which receive a proportion of funding from the NIHR Biomedical Research Centre funding scheme. 


\section{Measurement of Liver and Spleen Interstitial Volume in Patients with Systemic Amyloid Light-Chain Amyloidosis using Equilibrium Contrast CT}

\section{$\underline{\text { Abstract }}$}

\section{Objectives:}

Investigate equilibrium contrast-enhanced CT (EQ-CT) measurement of extracellular volume fraction (ECV) in patients with systemic amyloid light-chain (AL) amyloidosis, testing the hypothesis that ECV becomes elevated in the liver and spleen and ECV correlates with other estimates of organ amyloid burden.

\section{Methods:}

26 patients with AL amyloidosis underwent EQ-CT and ECV was measured in the liver and spleen. Patients also underwent serum amyloid P (SAP) component scintigraphy with grading of liver and spleen involvement. Mann-Whitney $U$ test was used to test for a difference between patients with amyloid deposition (SAP grade 1-3) and those without (SAP grade 0). Variation in ECV across SAP grades was assessed using the Kruskal-Wallis test and association between ECV and SAP grades with Spearman correlation.

\section{Results:}

Mean ECV in the spleen and liver were significantly greater $(\mathrm{p}<0.0005)$ in amyloidotic organs (SAP grade 1-3) [spleen, liver: 0.430, 0.375] compared with healthy tissues [spleen, liver: $0.304,0.269]$. ECV increased with increasing amyloid burden, showing positive correlation with SAP grade in both the liver $(r=0.758)$ and spleen $(r=0.867)$.

\section{Conclusion:}

In patients with systemic AL amyloidosis, EQ-CT can demonstrate increased spleen and liver $\mathrm{ECV}$, which is associated with amyloid disease burden. 


\section{$\underline{\text { Keywords }}$}

Tomography, X-ray Computed; Contrast Media; Amyloidosis; Radiographic Image Interpretation, Computer Assisted; Extracellular Space

\section{Abbreviations and Acronyms:}
AL Amyloidosis: Systemic amyloid light-chain amyloidosis EQ-CT: Equilibrium contrast-enhanced computed tomography EQ-MRI: Equilibrium contrast-enhanced magnetic resonance imaging EQ-CMR: Equilibrium contrast cardiac MRI ECV: Extracellular volume fraction SAP: Serum amyloid P 


\section{Introduction}

Amyloidosis is caused by the extracellular deposition of misfolded aggregated protein material, leading to organ dysfunction. Systemic amyloidosis is thought to be responsible for approximately one in 1,500 deaths per year in the UK [1]. Primary systemic light chain amyloidosis (AL amyloidosis), the most common form worldwide, commonly involves gastrointestinal organs, including the liver (62-90\%) and spleen (83\%) [2]. In hepatic amyloidosis, amyloid is deposited along the sinusoids and blood vessel walls, inhibiting diffusion and causing hepatocyte compression. Hepatic involvement may lead to painful hepatomegaly, liver failure, portal hypertension, rupture and haemorrhage. Amyloid affecting the spleen tends to infiltrate and deposit in the red pulp and blood vessel walls causing splenomegaly, and occasionally spontaneous rupture or haemorrhage [3,4].

Histological analysis is the reference standard test for diagnosis of amyloid, but it has various shortcomings. It is invasive, carries risk of complications, and is prone to sampling error. This makes it unsuitable for evaluating the wider distribution of amyloid or disease progression [5]. Currently the only non-invasive method of assessing amyloid burden is serum amyloid component P component (SAP) scintigraphy [5]. It is a very effective method for identifying and measuring amyloid in large solid organs, especially the liver and spleen, which have a fenestrated epithelium that allows efficient localisation of this large molecule. However, this radionucleotide is costly and not commercially available. There are only two centres in the world (London, UK, and Groningen, the Netherlands) [5] which currently use this technique routinely. There is otherwise no conventional imaging method to reliably diagnose amyloid deposition in the liver and spleen. Liver and spleen size measurements are commonly used to suggest deposition but this is neither specific nor reliable.

The emergence of equilibrium contrast enhanced imaging using computed tomography (EQCT) and magnetic resonance imaging (EQ-MRI) has provided a powerful new tool for assessing disorders that result in interstitial expansion including myocardial and liver fibrosis, cirrhosis and amyloidosis [6-13]. Extracellular volume fraction (ECV) quantified by equilibrium contrast cardiac MRI (EQ-CMR) and EQ-CT can accurately quantify amyloid burden in the myocardium when compared with current standard of care tests for amyloid [7,8]. EQ-MRI has already demonstrated accurate quantification of amyloid burden in the liver and spleen with validation 
against SAP scintigraphy [14]. However, use of the cheaper and potentially more widely available EQ-CT technique has yet to be assessed.

The purpose of our study was to test the hypothesis that ECV measured using EQ-CT increases with amyloid involvement in the liver and spleen, and to validate these results against the reference standard test of SAP scintigraphy for measuring organ amyloid load. 


\section{Materials and Methods}

Local ethics committee approval was obtained and all participants were provided informed written consent.

\section{Clinical Evaluation:}

Patients with AL amyloidosis were recruited from the outpatient clinic at the National Amyloidosis Centre in London. Inclusion criteria were biopsy proven AL amyloidosis demonstrated on Congo red and immune-specific staining. Biopsies were from various sites of the body including the kidney, heart, liver or other soft tissues, and the site of choice was directed by the clinical presentation of the individual. Patients with impaired renal function (estimated glomerular filtration rate $<40 \mathrm{ml} / \mathrm{min}$ ) and any standard contraindication to CT imaging were excluded.

Recruitment took place from January 2013 to August 2015.

\section{Equilibrium CT Imaging to measure ECV:}

The principals of equilibrium imaging have been described in prior work $[9,10]$. In brief, iodinated contrast is an extra-vascular, extra-cellular tracer. After intravenous delivery and sufficient time for equilibration, it partitions in water between cells in blood (plasma) and tissue (interstitial fluid). The tissue interstitial volume is elevated in the setting of fibrosis, amyloid and oedema. The ratio of signal change before and after contrast in blood and tissue is the partition coefficient (ratio of volumes of distributions). If the plasma volume is substituted (1-haematocrit), the tissue extracellular volume (ECV) is obtained.

CT examinations were performed (Aquilion ONE - Toshiba Medical Systems Corporation, Otawara-shi, Japan or Somatom Sensation 64 - Siemens Medical Solutions, Erlangen, Germany) using the following protocol. Prior to imaging, a 5-ml blood sample was taken from each patient and sent to the laboratory to measure blood haematocrit. Patients underwent a precontrast CT scan to obtain baseline blood, liver and spleen attenuation in Hounsfield units (HU). The iodinated contrast agent ioxhexol (Omnipaque 300; General Electric Healthcare, Cork, Ireland) was administered as a bolus dose $(1 \mathrm{ml} / \mathrm{kg}$ at $3 \mathrm{ml} / \mathrm{sec})$ without a saline chaser. 
As established in previous work, there was a five minute delay to allow for dynamic blood and organ contrast equilibration before a repeat scan was performed to measure blood, liver and spleen attenuation [9]. Refer to Table 1 for details of scan parameters.

Image analysis was performed using open source software (OsiriX Foundation, Geneva, Switzerland) by a radiologist (10 years of experience interpreting body CT scans) who was blinded to SAP results.

Slices through the mid portion of each organ where manually selected demonstrating the greatest cross-sectional area. In the liver, a peripherally based wedge-shaped region of interest (ROI) was drawn in the right lobe of the liver (mean area, $35.6 \mathrm{~cm}^{2}$ ), avoiding visible vessels or incidental hepatic lesions, whilst including the greatest area of liver parenchyma. Similar care was taken with the elliptical ROI drawn in the spleen (mean area, $11.1 \mathrm{~cm}^{2}$ ). Elliptical ROI were also drawn within the abdominal aorta (mean area, $2.3 \mathrm{~cm}^{2}$ ), with care taken to avoid the aortic wall and any atheromatous plaques. In the liver and spleen, ROI were drawn in three consecutive axial slices to obtain an average attenuation. In the aorta, this was performed in 15 consecutive slices, with more measurements taken to account for the greater inter-slice variation in attenuation due to the effects of beam hardening (due to the closer proximity of the vertebral endplates to the aorta).

The ROI were drawn on the images obtained after contrast agent administration, before being copied to a visually matched pre-contrast image (Fig 1). If an organ was incompletely imaged on either phase or if artefacts degraded the image significantly, the data for the organ in that individual was excluded. Mean attenuation (in Hounsfield units) for liver parenchyma, splenic parenchyma and blood were derived and liver and spleen ECV were calculated using the following formula:

$E C V_{\text {liver }}=(1-$ hematocrit $) \times\left(\Delta H U_{\text {liver }} / \Delta H U_{\text {blood }}\right)$,

where $\Delta H U$ denotes Hounsfield units in equilibrium phase minus Hounsfield units before contrast agent administration.

\section{SAP Scintigraphy:}

Patients with AL amyloidosis underwent scintigraphy with iodine 123 labelled SAP (123I-SAP) as part of their routine assessment [15]. SAP imaging was performed within four weeks of CT 
imaging. Anterior and posterior whole body images were acquired using a GE Infinia gamma camera (General Electric, Milwaukee, Wisconsin) and medium-energy collimator 24 hours after administering $200 \mathrm{MBq}{ }^{123}$ I-SAP.

A single physician (with experience of more than 30,000 SAP imaging studies) graded the total body liver and splenic amyloid burden by visual assessment ( 0 , no involvement; 1 , small; 2 , moderate; 3, large) [16]. Patients with a congestive pattern of activity in the spleen or liver on SAP scintigraphy had the affected organ excluded from analysis, as congestion prevented meaningful SAP grading.

\section{Statistical Analysis:}

Statistical analysis was performed using software (Prism 5.0; Graphpad, San Diego, CA, USA). Spearman correlation analysis was used to establish an association between ECV and SAP grades in the liver and spleen, with one-sided significant testing (set to $\mathrm{P} \leq 0.05$ ). ECV and SAP grades were compared by using Kruskal-Wallis non-parametric analysis of variance test, with Dunn's multiple comparison test performed after to analyse significance between ECV values in the different SAP grades. Mann-Whitney U test was used to compare ECV values between healthy and diseased tissue (liver and spleen with a SAP grade between one and three). 


\section{$\underline{\text { Results }}$}

42 patients were identified satisfying the inclusion criteria. 16 patients were excluded due to impaired renal function (eGFR<40ml/min). 26 patients ( 11 women and 15 men; median age, 63 years; age range, 41-75) underwent EQ-CT assessment of their abdomen, with SAP scintigraphy as part of their routine clinical care. One patient was excluded due to congestive hepatosplenomegaly and two patients because of congestive hepatomegaly on SAP scintigraphy. Therefore, paired SAP and ECV data were available for the liver in 23 patients, and for the spleen in 24 patients (Table 2).

The mean attenuation in the liver, spleen and aorta pre-contrast were 56.3 HU (range 34.3 66.4 HU), 47.6 HU (range 30.1- 66.4 HU) and 42.7 HU (range 31.2 - $69.0 \mathrm{HU}$ ) respectively; and at equilibrium phase were $68.5 \mathrm{HU}$ (range 49.5 - 82.6 HU), $62.1 \mathrm{HU}$ (range 43.0 - $83.1 \mathrm{HU}$ ) and 67.7 HU (range 52.3 - 81.9 HU) respectively. The mean total calculated effective radiation dose for the EQ-CT protocol on the Siemens scanner was $7.03 \mathrm{mSv}+/-1.73 \mathrm{mSv}$, and $3.14 \mathrm{mSv}+/$ $1.20 \mathrm{mSv}$ on the later generation Toshiba scanner.

In healthy tissue (liver and spleen with a SAP grade of zero), the median liver ECV was 0.265 (interquartile range [IQR], 0.242 - 0.289) and the median spleen ECV was 0.299 (IQR, 0.281 0315). In disease (liver and spleen with a SAP grade between one and three), the median liver ECV was 0.387 (IQR, 0.346 - 0.427) and the median spleen ECV was 0.433 (IQR, $0.365-0.500$ ). In disease, ECV in both organs were significantly elevated compared to the healthy group [SAP grade 0] $(\mathrm{p}<0.005)$ [figure 2]. ECV increased with SAP grade in both organs with positive correlation in both the liver $(r=0.758,95 \%$ confidence interval $0.493-0.895)$ and spleen $(r=$ $0.867,95 \%$ confidence interval $0.707-0.943$ ) [figure 3]. 


\section{Discussion}

In this exploratory study, EQ-CT was used to measure ECV in the liver and spleen of patients with systemic AL amyloidosis. We found that EQ-CT could differentiate healthy liver or spleen from organs infiltrated by amyloid, and that ECV tracked disease burden. The association of ECV with SAP grade suggests that elevated ECV reflects underlying amyloid deposition. This is consistent with other studies using EQ-MRI in the heart, liver and spleen $[8,14]$ and with EQ-CT assessment of cardiac amyloidosis [9].

By developing the EQ-CT method, several of the advantages of CT over MRI can be exploited. These include cost, fewer contraindications and simpler contrast-signal relationship.

We compared EQ-CT derived ECV with SAP grading for each organ. SAP grading is a wellvalidated scoring system to visually grade amyloid burden in AA (amyloid A protein) and AL amyloidosis [17], with high diagnostic sensitivity and specificity. However, there are limitations of SAP scintigraphy [18]. The subjective visual grading system is prone to interoperator variability, and requires highly specialist expertise. ${ }^{123} \mathrm{I}$ is also costly and SAP is not readily available. EQ-CT is also advantageous in that it allows objective and quantitative measurement of amyloid burden, with the ability to evaluate regional variations in expansion of the extracellular space.

Study limitations include the patient selection method, which resulted in a relatively high proportion of patients with a healthy liver and spleen. Although sufficient to demonstrate initial proof of principle, a future study should include a larger cohort of patients, distributed equally across the range of SAP grades to provide a more robust assessment of EQ-CT. Renal impairment (eGFR $<40 \mathrm{ml} / \mathrm{min}$ ), which we found in $38 \%$ of our screened population, also limited patient inclusion - as compared to $20 \%$ of the screened population in a similar EQMRI study [14] where a lower eGFR threshold of $30 \mathrm{ml} / \mathrm{min}$ applied. Renal function could limit the use of EQ-CT for assessment of more advanced systemic disease, where renal injury is common, but not so in early disease where there frequently is diagnostic uncertainty.

Another limitation to note is that SAP grading may not have a linear relationship with volume of amyloid deposition. Histological quantification of amyloidosis would provide a more robust 
standard of reference when comparing the relative accuracy of SAP grading and EQ-CT derived ECV in future studies.

The use of dynamic contrast equilibrium for ECV measurement in amyloidosis also has limitations. The shorter protocol may not allow sufficient time for contrast equilibration throughout the markedly expanded extracellular volume that occurs in advanced disease. White et al. showed using EQ-MRI and gadolinium in the heart that the bolus and infusion equilibrium methods provide equivalent estimations up to an extracellular volume fraction of 0.4 , but beyond this, the bolus technique overestimates ECV [19].

The EQ imaging method is non-specific measuring the tissue extracellular water fraction and other processes such as inflammation, venous congestion and fibrosis may cause an increase in the ECV. Risk factors for these processes were not assessed here. ECV measurement by equilibrium imaging also assumes a negligible intravascular volume within tissues, which in the liver and spleen may not be the case.

Standard CT image acquisition and reconstruction techniques were used in this study. With modern multi-detector scanners and reconstruction methods, image artefacts and noise are reduced, producing more precise and accurate ECV quantification at a lower dose of ionising radiation $[20,21]$. The mean size-specific dose estimate for spleen and liver equilibrium CT examination in this study was comparable to that of a standard clinical CT examination of the liver and was equivalent to approximately one year of natural background radiation in the United States for the later generation Toshiba scanner [22] (natural dose in the USA is 3.10 mSv per year).

Although the data displayed in figure 2 displays good separation between disease and healthy groups (suggesting high test sensitivity and specificity), the study sample was too small for meaningful receiver operating characteristic curve (ROC) analysis and we have not attempted to define a diagnostic cut-off. Single and multi-scanner ECV reproducibility and repeatability were also not assessed. In developing ECV as a potential diagnostic test, these are areas which require future evaluation. 
This initial study has shown that EQ-CT can identify and evaluate amyloid burden in the liver and spleen using widely available CT technology. EQ-CT then potentially offers a non-invasive tool for assessing key gastrointestinal organ involvement in patients with AL amyloidosis and has potential to aid diagnosis and treatment monitoring in the future. 


\section{Compliance with ethical standards}

\section{Informed consent:}

Informed consent was obtained from all individual participants included in the study.

\section{Ethical approval:}

Institutional Review Board approval was obtained.

All procedures performed in studies involving human participants were in accordance with the ethical standards of the Institutional Review Board and with the 1964 Helsinki declaration and its later amendments or comparable ethical standards. 


\section{$\underline{\text { References }}$}

1. Gillmore JD, Hawkins PN. Pathophysiology and Treatment of Systemic Amyloidosis. Nat Rev Nephrol. 2013; 9(10):574-586

2. Sattianavagam PT, Hawkins PN, Gillmore JD. Systemic Amyloidosis and the Gastrointestinal Tract. Nat Rev Gastroenterol Hepatol. 2009; 6(10):608-617

3. Lovat LB, Persey MR, Madhoo S, Pepys MB, Hawkins P. The Liver in Systemic Amyloidosis: Insights from 123I Serum Amyloid P Component Scintigraphy in 484 Patients. Gut 1998; 42(5):727-734

4. S Monzawa, Tsukamoto T, Omata K, Hosoda K, Araki T, Sugimura K. A Case with primary Amyloidosis of the liver and spleen: Radiologic Findings. Eur J Radiol 2002: 41(3):237241

5. Sachchithanantham S, Wechalekar AD. Imaging in Systemic Amyloidosis. Br Med Bull. 2013. 107(1):41-56

6. Georgiades CS, Neyman EG, Barish MA, Fishman EK. Amyloidosis: Review and CT Manifestations. Radiographics. 2004; 24(2):405-416

7. Bandula S, White SJ, Flett AS, et al. Measurement of Myocardial Extracellular Volume Fraction by using Equilibrium Contrast-enhanced CT: Validation Against Histologic Findings. Radiology. 2013; 269(2):396-403

8. Banypersad SM, Sado DM, Flett AS, et al. Quantification of Myocardial Extracellular Volume Fraction in Systemic AL Amyloidosis: An Equilibrium Contrast Cardiovascular Magnetic Resonance Study. Circ: Cardiovasc Imaging. 2013; 6(1):34-39

9. Treibel TA, Bandula S, Fontana M, et al. Extracellular Volume Quantification by Dynamic Equilibrium Cardiac Computed Tomography in Cardiac Amyloidosis. J Cardiovasc Comput Tomogr. 2015; 9(6):585-592 
10. Bandula S, Punwani S, Rosenberg WM, et al. Equilibrium Contrast-enhanced CT Imaging to Evaluate Hepatic Fibrosis: Initial Validation by Comparison with Histopathologic Sampling. Radiology. 2015; 275(1)

11. Varenika V, Fu Y, Maher JJ, Gao D, Kakar S, Cabarrus MC, Yeh BM. Hepatic fibrosis: evaluation with semiquantitative contrast-enhanced CT. Radiology. 2013; 266(1):151-8

12. Zissen MH, Wang ZJ, Yee J, Aslam R, Monto A, Yeh BM. Contrast-enhanced CT quantification of hepatic fractional extraceullar space: correlation with diffuse liver disease severity. AJR Am J Roentgenol. 2013; 201(6):1204-10

13. Yoon JH, Lee JM, Klotz E, Jeon JH, Lee KB, Han JK, Choi BI. Estimation of hepatic extracellular volume fraction using multiphasic liver computed tomography for hepatic fibrosis grading. Invest Radiol. 2015;50(4):290-6

14. Bandula S, Banypersad SM, Sado DM, et al. Measurement of Tissue Interstitial Volume in Healthy Patients and Those With Amyloidosis with Equilibrium Contrast-enhanced MR Imaging. Radiology. 2013; 268(3):858-864

15. Hawkins PN, Lavender JP, Pepys MB. Evaluation of Systemic Amyloidosis by Scintigraphy with 123 I-labelled Serum Amyloid P Component. N Engl J Med. 1990; 25(7):709-713

16. Rydh A, Suhr O, Hietala SO, Ahlström KR, Pepys MB, Hawkins PN. Serum Amyloid P Component Scintigraphy in Familial Amyloid Polyneuropathy: Regression of Visceral Amyloid Following Liver Transplantation. Eur J Nucl Med. 1998; 25(7):2197-2215

17. Sachchithanantham S, Wechalekar AD. Imaging in Systemic Amyloidosis. Br Med Bull. 2013. 107(1):41-56 
18. Hazenburg BP, van Rijswijk MH, Piers DA, Lub-de Hooge MN, Vellenga E, Haagsma EB, Hawkins PN, Jager PL. Diagnostic Performance of 123I-Labeled Serum Amyloid P Component Scintigraphy in Patients with Amyloidosis. Am J Med. 2006; 119(4):355

19. White SK, Sado DM, Fontana M, et al. T1 mapping for myocardial extracellular volume measurement by CMR: bolus only versus primed infusion technique. JACC Cardiovasc Imaging. 2013; 6(9):955-62

20. Chen B, Marin D, Richard S, Husarik D, Nelson R, Samei E. Precision of Iodine Quantification in Hepatic CT: Effects of Iterative Reconstruction with Various Imaging Parameters. AJR Am J Roentgenol. 2013; 200(5):W475-W482

21. Shuman WP, Green DE, Busey JM, et al. Model-based Iterative Reconstruction Versus Adaptive Statistical Iterative Reconstruction and Filtered Back Projection in Liver 64MDCT: Focal Lesion Detection, Lesion Conspicuity and Image Noise. AJR AM J Roentgenol. 2013; 200(5):1071-1076

22. Kase KR, Strom DJ, Thomadsen BR, Suleiman OH, Quinn DM, Miller KL. National Council on Radiation Protection Report no. 160, Ionizing Radiation Exposure of the Population of the United States. National Council on Radiation Protection and Measurements. 2009. 


\section{$\underline{\text { Illustrations and Tables }}$}

Table 1. EQ-CT scan parameters.

Figure 1. EQ-CT images pre and at EQ contrast phase through the upper abdomen showing example regions of interest drawn in the liver, spleen and abdominal aorta.

Table 2. Number of cases of patients with a given SAP grade in the liver and spleen.

Figure 2. Box and whisker plots of ECV, with individual data points, in healthy versus diseased tissue in a) liver $(\mathrm{p}<0.0005)$ and (b) spleen $(\mathrm{p}<0.0001){ }^{* * *} \mathrm{p}=0.0001-.001,{ }^{* * * *} \mathrm{p}=<0.0001$.

Figure 3. Box and whisker plots of ECV (median and standard deviation) by SAP grade in the (a) liver ( $\mathrm{p}<0.005, \mathrm{r}=0.758$ ) and (b) spleen ( $<<0.001, \mathrm{r}=0.867)$ demonstrating increasing ECV with SAP. ${ }^{*} \mathrm{p}=0.01-.05$. n.s. $=$ not significant $(\mathrm{p}>0.05)$ 


\section{Pre-Contrast}

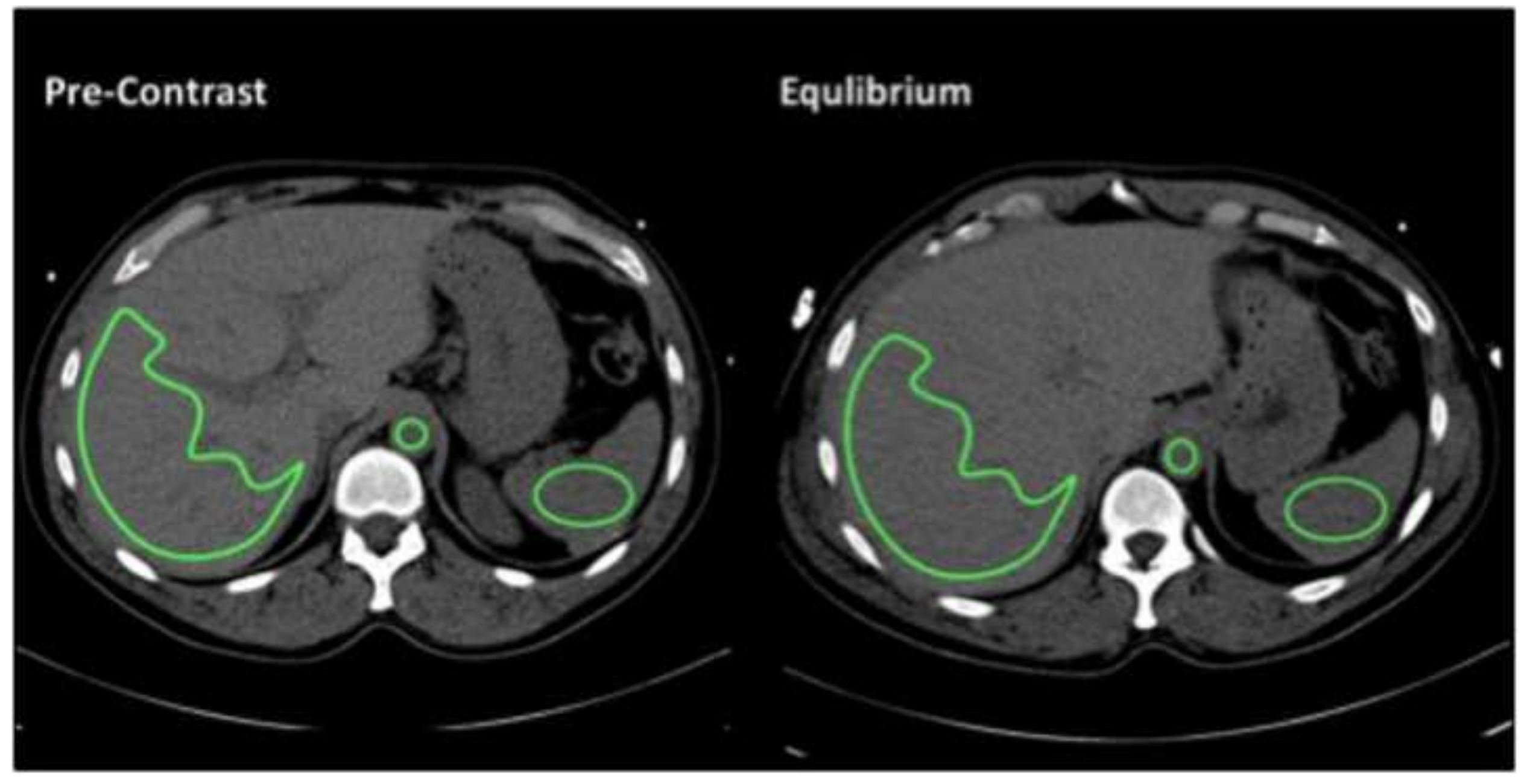

\section{Equlibrium}

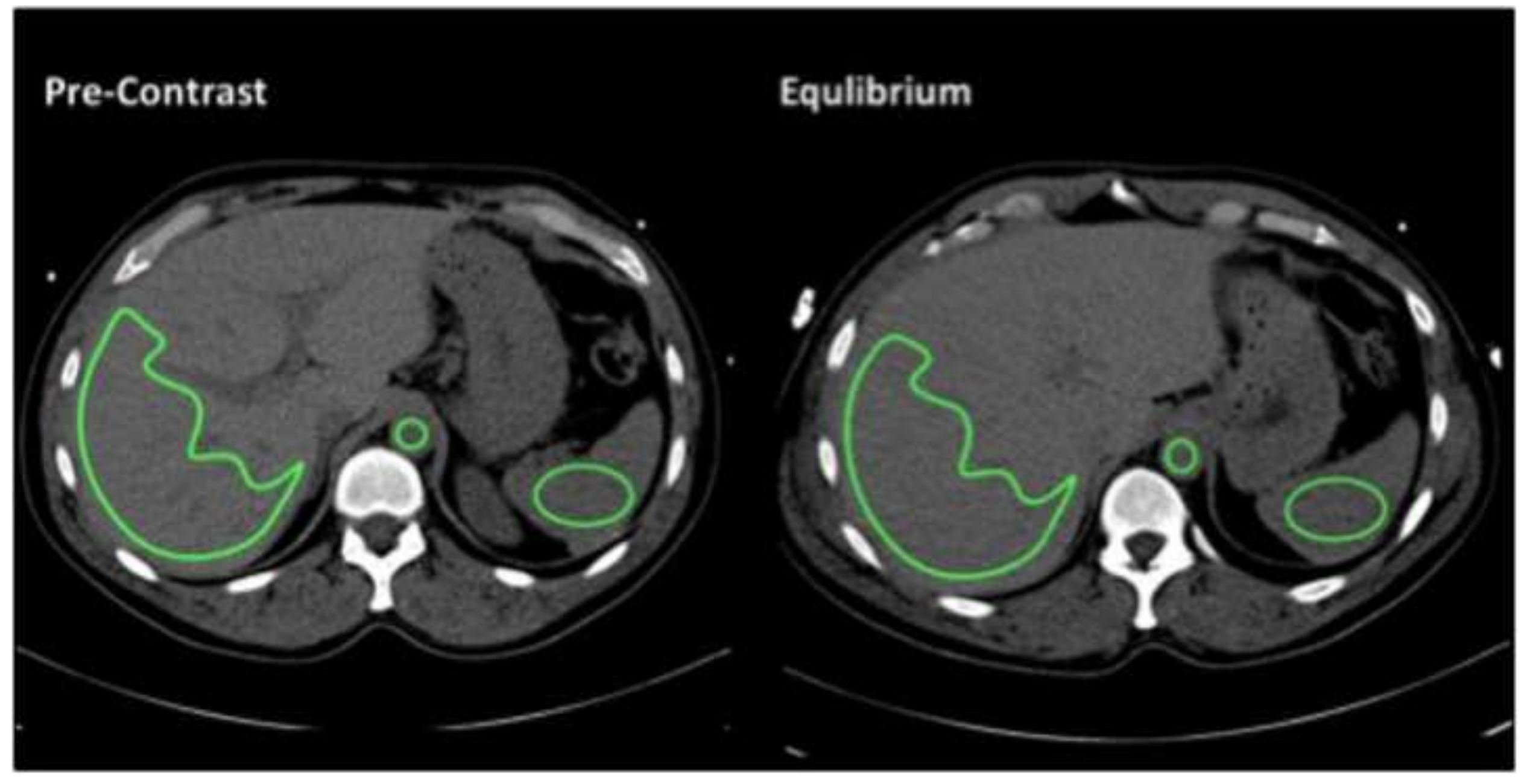



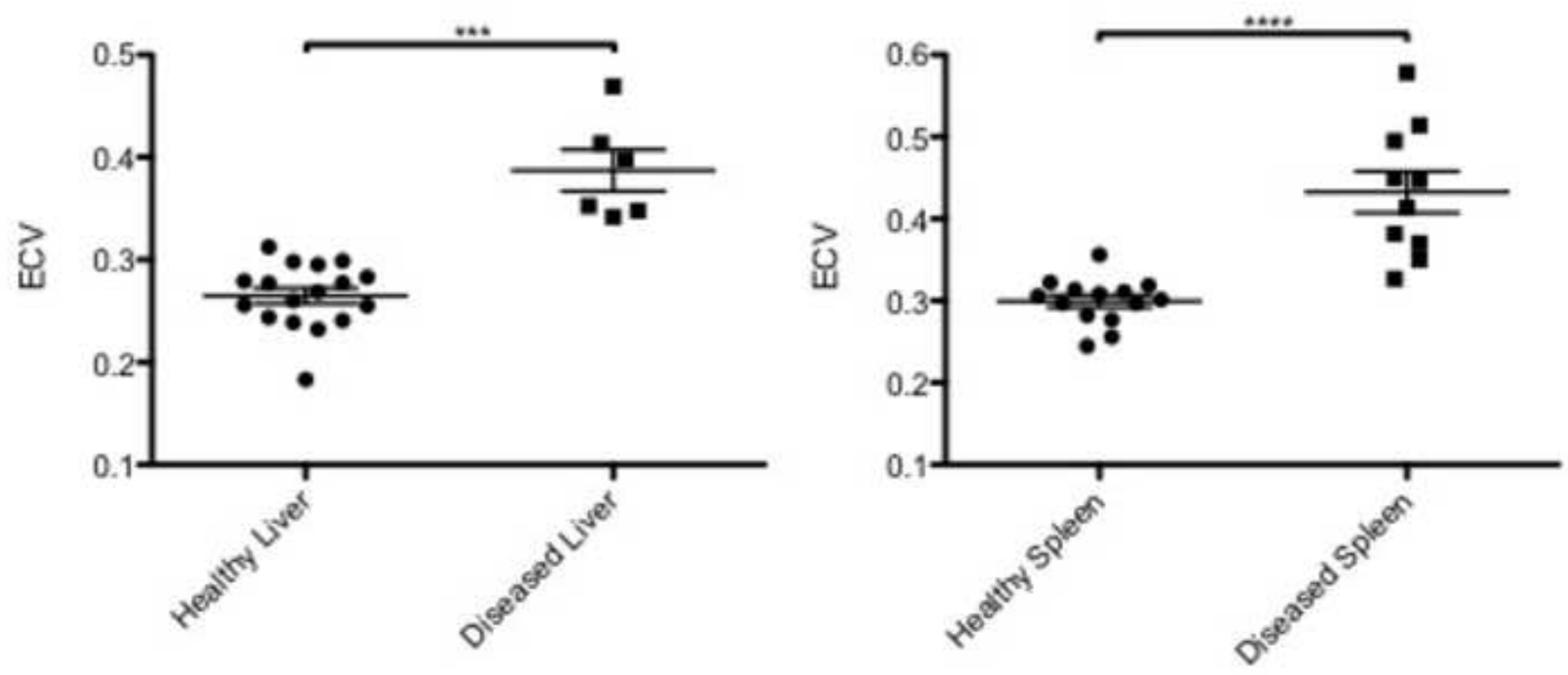

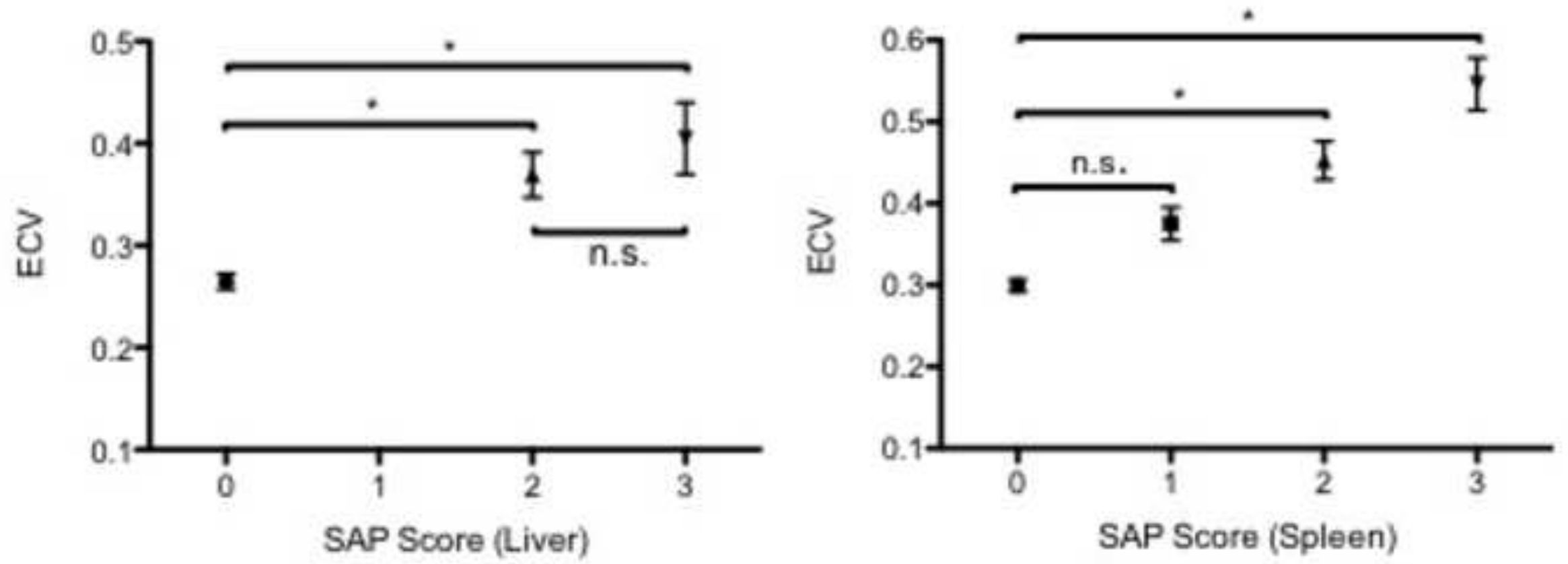
Siemens

Tube Current (mA)

Tube Voltage (kV)

Section Collimation

Section Thickness ( $\mathrm{mm}$ )

Gantry Rotation Time (ms)

Convolution Kernel
Modulated

120

64 detector rows

1.2

500

B20f (soft tissue)
Toshiba

Modulated

120

320 detector rows

0.5

350

FCO7-H (body standard volume) 


\section{SAP Grade \\ Number of Patients}

\section{Liver}

\section{Spleen}

\begin{tabular}{c|c}
17 & 14 \\
0 & 5
\end{tabular}

$3 \quad 3$

3

3

2

Total

23

24 